\title{
A controlled study of potential risk factors preceding exacerbation in multiple sclerosis
}

\author{
C Gasperini, M G Grasso, M Fiorelli, E Millefiorini, S Morino, A Anzini, A Colleluori, \\ M Salvetti, C Buttinelli, C Pozzilli
}

\begin{abstract}
A wide variety of potential risk factors for acute exacerbations in multiple sclerosis were evaluated in a one year case-control study. Eighty nine consecutive patients with clinically definite multiple sclerosis and relapsing remitting course presenting with a relapse between January and December 1992 were compared with patients matched for age, sex, and degree of disability, who did not experience clinical exacerbations during the same period. Only potential risk factors occurring in the three months preceding the interview were considered patients. Relapsing patients reported no significant increase in the frequency of any risk factor in the three month period before exacerbation compared with the control group. These results suggest that most relapses are not preceded by the conditions commonly considered as risk factors.
\end{abstract}

(F Neurol Neurosurg Psychiatry 1995;59:303-305)

Keywords: exacerbation; multiple sclerosis; risk factors

Because the aetiology of multiple sclerosis is still unknown, the onset (or relapses) of the disease has been associated with many factors including age, sex, latitude, migration, geographic location, race, familiar aggregation, and viral exposure. ${ }^{1}$ Among factors which may trigger exacerbations of multiple sclerosis the more commonly reported were viral infections, ${ }^{2-4}$ vaccinations, ${ }^{56}$ trauma, ${ }^{7}$ anaesthesia, ${ }^{8}$ physical overexertion, ${ }^{9}$ purperium, ${ }^{1011}$ season, ${ }^{9}$ and stressful life circumstances. ${ }^{12} 13$ In what proportion relapses in multiple sclerosis are triggered by an identifiable factor is still unsettled.

To investigate the role of potential risk factors in acute exacerbations, we designed a controlled one year study in patients with relapsing and remitting multiple sclerosis.

\section{Materials and methods}

Of patients presenting to the multiple sclerosis Centre of the University of Rome "La Sapienza" between 1 January and 31
December 1992, we focused our attention on those with definite or laboratory supported multiple sclerosis according to Poser criteria. Case patients were all those with a known exacerbating and remitting course who came to us with their first relapse of the year. An exacerbation was defined as a new neurological symptom, associated with a change of at least one point in expanded disability status score (EDSS) which lasted more than 24 hours. Patients found to have a progressive course or severe cognitive impairment were not eligible for the study. Controls were selected from patients without clinical exacerbation who were referred to our centre during the same period for routine clinical examination. They also had an exacerbating and remitting multiple sclerosis, but with a stable course in the past three months. Controls were interviewed during the same study period. One control was matched to each case by sex, age within three years, EDSS score within 0.5 points, and season in which the interview took place.

\section{DATA COLLECTION}

Information on previous exposures was collected during a structured interview, which systematically took place at the end of the visit, after the EDSS. The interview was carried out by AA or AC, who are consultant neurologists with a special interest in multiple sclerosis. The purpose of the study was explained to participants before starting the interview. Questions were asked in a simple and comprehensible manner. The duration was of about 15 minutes. Patients were questioned about their exposure to the following putative risk factors during the preceding three months:

Infectious disease-Limited to influenza, upper respiratory syndromes, gastroenteritis, and bacterial urinary tract infections.

Stressful life circumstances-Any life event which produced emotional tension distinctly beyond the usual tensions of everyday life. Such life events were classified into nine subcategories according to the method of Sibley ${ }^{9}$ (table 1) and did not include events which patients did not consider stressful.

Trauma-Head or back trauma, ranging from mild contusion to fractures.

Physical overexertion-Any unusual physical exertion, usually consisting of such matters as 
Table 1 Classification of stressful events

\begin{tabular}{ll}
\hline I & Death of spouse or close relative \\
II & Death of other close family member \\
III & Marital stress: infidelity, conflict, separation, divorce \\
IV & Job loss or severe work stress \\
V & Job loss: spouse \\
VI & Personal illness: (other than multiple sclerosis), trauma, surgery \\
VII & Serious illness: family member \\
VIII & Interpersonal conflict: family or others; includes family financial problems \\
IX & Miscellaneous: friends' or pets' death; thefts; lawsuits, etc \\
\hline
\end{tabular}

From Sibley $1962^{2}$.
(6.8) (range 1-36) years, and 1.5 (1.0) (range $0-4)$ for the control group.

Overall, 59 cases and 50 controls reported an exposure to one or more potential risk factors in the preceding three months. Table 2 shows the occurrence of selected and total risk factors for both cases and controls. No significant differences were found between cases and controls for a single stressful life circumstance; cumulated stressful events were associated with a higher risk for subsequent exacerbation but this did not reach significance. A significantly higher percentage of controls (11) than cases (2) reported having had exposure to sudden environmental temperature variation. For the remaining risk factors we did not find any significant differences. Exposure mental temperature variation.

Vaccination-Limited to hepatitis, influenza, rubella, tetanus, tuberculosis, and typhus.

Exposure to toxins-Any occupational exposure, with particular regard to white spirit, petroleum spirit, petroleum, and metals. Five questions were devoted to medical care, particularly the use of drugs, history of radiotherapy, or radiographic examinations.

Anaesthesia-General anaesthesia, local and regional anaesthesia (epidural and spinal).

DATA ANALYSIS

The effect of exposures was assessed by computing matched odds ratios with their corresponding $95 \%$ confidence intervals (95\% CIs).

\section{Results}

The study group comprised 89 case-control pairs. Sixty two were women and 27 were men. The mean (SD) age, disease duration, and EDSS were respectively $35 \cdot 6(9 \cdot 3)$ (range 18-56) years, $7 \cdot 4(6 \cdot 1)$ (range 1-36) years, and $1.7(0.8)$ (range $0-4.5)$ for the case group and $35.3(10 \cdot 1)$ (range 18-58) years, 7.9

Table 2 Reported occurrence of selected risk factors

\begin{tabular}{lccl}
\hline & $\begin{array}{c}\text { Cases } \\
(n=89)\end{array}$ & $\begin{array}{c}\text { Controls } \\
(n=89)\end{array}$ & $\begin{array}{l}\text { Odds ratio } \\
(95 \% \mathrm{CI})\end{array}$ \\
\hline Infectious diseases: & 4 & 8 & $0 \cdot 5(0 \cdot 2-1 \cdot 7)$ \\
$\quad$ Influenza & 2 & 2 & $1 \cdot 0(0 \cdot 1-7 \cdot 3)$ \\
Upper respiratory infectious & 0 & 0 & Undefined \\
Exanthematous disease & 0 & 1 & 0 \\
Diarrhoea & 0 & 2 & 0 \\
Urinary infections & 6 & 13 & $0 \cdot 5(0 \cdot 2-1 \cdot 3)$ \\
Total infectious diseases & 3 & 1 & $3 \cdot 0(0 \cdot 3-29 \cdot 8)$ \\
Stressful life events: & 1 & 0 & Undefined \\
Type I & 0 & 0 & Undefined \\
Type II & 1 & 2 & $0 \cdot 5(0 \cdot 4-5 \cdot 7)$ \\
Type III & 4 & 6 & $0 \cdot 6(0 \cdot 2-2 \cdot 4)$ \\
Type IV & 0 & 0 & Undefined \\
Type V & 5 & 1 & $5 \cdot 0(0 \cdot 6-44 \cdot 1)$ \\
Type VI & 6 & 1 & $6 \cdot 0(0 \cdot 7-51 \cdot 3)$ \\
Type VII & 2 & 1 & $2 \cdot 0(0 \cdot 2-22 \cdot 8)$ \\
Type VIII & 22 & 12 & $2 \cdot 3(0 \cdot 9-5 \cdot 6)$ \\
Type IX & 0 & 3 & 0 \\
Total stressful circumstances & 0 & 3 & 0 \\
Trauma & 2 & 11 & $0 \cdot 2(0 \cdot 04-0 \cdot 8)$ \\
Physical overexertion & 0 & 6 & 0 \\
Temperature variation & 0 & 1 & 0 \\
Vaccination & 0 & 2 & 0 \\
Exposure to toxins & 30 & 39 & $0 \cdot 6(0 \cdot 3-1 \cdot 2)$ \\
Anaesthesia & & & \\
Patients with one or more risk factors & & & \\
\hline
\end{tabular}

\section{Discussion}

We were unable to identify any candidate risk factor that may trigger exacerbations in multiple sclerosis. The low frequency of some risk factors in this study, such as trauma, physical overexertion, anaesthesia, and exposure to toxins, may have limited the capability to uncover potential weak associations between risk factors and relapses.

There is a lack of consensus about the effect of trauma on the exacerbation and the progression of the disease. McAlpine et $a l^{14}$ and Walton ${ }^{15}$ favoured the hypothesis that trauma must be considered a precipitating factor for exacerbation. In accord with other studies, ${ }^{716}$ we did not find a relation between traumatic episodes and most forms of physical trauma. Our data confirm previous studies which have not provided evidence that physical overexertion ${ }^{9}$ or anaesthesia ${ }^{8}$ are risk factors for disease exacerbation.

The possibility that vaccinations increase the risk of relapses in multiple sclerosis is debated. Riikonen ${ }^{17}$ showed that the common vaccinations with live or inactivated viruses were often preceding events in relapses of multiple sclerosis. Bamford et $a l^{5}$ and more recently Salvetti et $a l^{6}$ showed a lower incidence of relapse in patients with multiple sclerosis treated with swine influenza vaccination and concluded that the use of a vaccine does not seem to influence the course of disease.

The role of stressful life circumstances on onset and exacerbations of multiple sclerosis was first studied by Pratt. He did not find a statistically significant difference between patients with multiple sclerosis and a nonmatched control group in their experience of who reported significant stressful events were at a significantly higher risk of relapses. ${ }^{12} \mathrm{By}$ contrast the results obtained by Sibley ${ }^{3}$ and more recently Nisipeanu and Korczyn, ${ }^{13}$ did not support the influence of stress on the course of multiple sclerosis. In our study no significant differences were found between cases and controls for a single stressful life circumstance; cumulated stressful events were associated with a higher risk for life events. ${ }^{18}$ Franklin et al found that patients 
subsequent exacerbation but it did not reach significance.

By far the strongest environmental risk factors identified were virus-like infections. Sibley ${ }^{3}$ and Andersen et $a l^{4}$ found that in at risk periods-that is, from two weeks before an upper respiratory infection to five weeks afterwards, the multiple sclerosis exacerbation rate was higher than during not at risk periods. Panitch, ${ }^{19}$ recently, in a prospective study on viral infections and their correlation with multiple sclerosis attacks, found a strong correlation between multiple sclerosis exacerbations and upper respiratory infections. The lack of a relation between viral infections and exacerbations in our study can be explained by the different methodological approach used. Previous reports evaluated the relative risk of the exacerbations as annual relapse frequencies both in at risk and not at risk periods whereas our case-control study considered the association of a relapse with a viral infection verified in the previous three months.

Another concern in our study was the selection of cases and controls based only on clinical grounds. It is known that MRI in multiple sclerosis is more sensitive than clinical evaluation in detecting disease activity; new and enlarging lesions have often been seen in apparently clinically stable patients. ${ }^{20}$ Therefore, it may be possible that in some patients of our control group, the disease was active biologically but not clinically detectable.

In conclusion, the most important indication resulting from this study is the lack of strong relations between potential risk factors and exacerbations. Our data suggest a reassuring message for patients with multiple sclerosis. They should lead their lives without many of the restrictions advised by physicians until now.
We thank Professor Cesare Fieschi, Department of Neurological Science, University of Rome "La Sapienza" for his helpful comments.

1 Kurtzke JF. Epidemiological evidence for multiple sclerosis as an infection. Clin Microbiol Rev 1993;6:382-427.

2 Sibley WA. Risk factors in multiple sclerosis-implications for pathogenesis. In: Serlupi Crescenzi $G$, ed. Multidisciplinary approach to myelin disease. Life Science Vol 142. New York: Plenum Press, 1986

3 Sibley WA, Bamford CR, Clark K. Clinical viral infections and multiple sclerosis. Lancet 1985;i:1313-5.

4 Andersen $O$, Lygner PE, Bergstrom $T$, et al. Viral infections trigger multiple sclerosis relapses: a prospective seroepidemiological study. F Neurol 1993;240:417-22.

5 Bamford CR, Sibley WA, Laguna JF. Swine influenza vaccination in patients with multiple sclerosis. Arch Neurol 1978;35:242-3.

6 Salvetti M, Pisani A, Bastianello S, et al. Clinical and MRI assessment of disease activity in patients with multiple sclerosis after influenza vaccination. $尹$ Neurol 1995;242:

Siva A, Radhakrishnan K, Kurland LT, et al. Trauma and multiple sclerosis: a population-based cohort study from Olmsted County Minnesota. Neurology 1993;43: 1878-82.

8 Bamford CR, Sibley WA, Laguna JF. Anesthesia in multi-

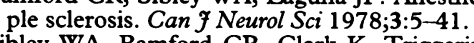

9 Sibley WA, Bamford CR, Clark K. Triggering factors in multiple sclerosis. In: Poser CM, ed. The diagnosis of multiple sclerosis. New York: Thieme-Stratton Inc, Georg Thieme Verlag. 1984:14-24.

10 Bernardi S, Grasso MG, Bertollini R, et al. The influence of pregnancy on relapses in multiple sclerosis: a cohort study. Acta Neurol Scand 1991;84:403-6.

11 Roullet E, Verdier-Taillefer MH, Amarenco P, et al. Pregnancy and multiple sclerosis: a longitudinal study of 125 remittent patients. $\mathcal{F}$ Neurol Neurosurg Psychiatry 125 remittent pati
1988;51:495-8.

12 Franklin GM, Nelson LM, Heaton RK, et al. Stress and its relationship to acute exacerbations in multiple scleroits relationship to acute exacerba
sis. $\mathcal{F}$ Neurol Rehab $1988 ; 2: 7-11$.

13 Nisipeanu P, Korczyn AD. Psychological stress as risk factor for exacerbations in multiple sclerosis. Neurology 1993;43:1311-3.

14 McAlpine D, Lumsden CE, Acheson ED. Multiple sclerosis-a reappraisal. Edinburgh: Churchill Livingstone, 1968:72.

15 Walton J. Brain's diseases of the nervous system 8th ed. Oxford: Oxford University Press, 1977:547-8.

16 Kurland LT. Trauma and multiple sclerosis. Ann Neurol 1994;36:S33-7.

17 Riikonen $R$. The role of infection and vaccination in the genesis of optic neuritis and multiple sclerosis in genesis of optic neuritis and multiple
children. Acta Neurol Scand 1989;80:425-31.

18 Pratt R. An investigation of the psychiatric aspects of disseminated sclerosis. F Neurol Neurosurg Psychiatry 1951; 14:326-36.

19 Panitch HS. Influence of infection on exacerbation of multiple sclerosis. Ann Neurol 1994;36:S25-8.

20 Bastianello S, Pozzilli C, Bernardi S, et al. Serial study of gadolinium-DPTA MRI enhancement in multiple sclerosis. Neurology 1990;40:591-5. 